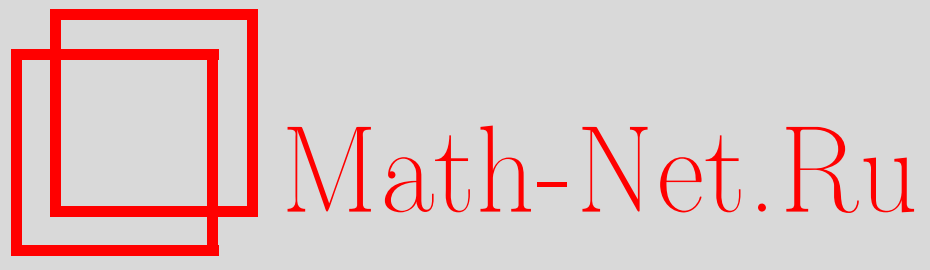

S. Blei, H.-J. Engelbert, A note on one-dimensional stochastic differential equations with generalized drift, Теория вероятн. и ее примен., 2013, том 58, выпуск 3, 506-520

DOI: https://doi.org/10.4213/tvp4523

Использование Общероссийского математического портала Math-Net.Ru подразумевает, что вы прочитали и согласны с пользовательским соглашением http://www . mathnet.ru/rus/agreement

Параметры загрузки:

IP : 54.157 .27 .8

26 апреля 2023 г., 10:53:28

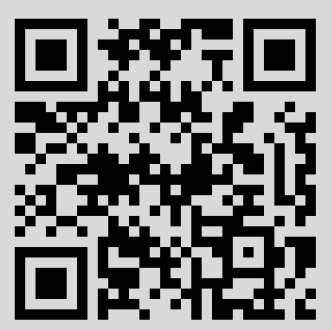




\section{A NOTE ON ONE-DIMENSIONAL STOCHASTIC DIFFERENTIAL EQUATIONS WITH GENERALIZED DRIFT ${ }^{1)}$}

Мы рассматриваем одномерные стохастические дифференциальные уравнения с обобщенным сносом, в которых присутствует локальное время $L^{X}$ процесса решения:

$$
X_{t}=X_{0}+\int_{0}^{t} b\left(X_{s}\right) \mathrm{d} B_{s}+\int_{\mathbf{R}} L^{X}(t, y) \nu(\mathrm{d} y),
$$

где $b$ - измеримая вещественная функция, $B$ - винеровский процесс и $\nu$ обозначает функцию множеств, определенную на ограниченных борелевских подмножествах вещественной оси $\mathbf{R}$ так, что она является конечной мерой со знаком на $\mathscr{B}([-N, N])$ для любого $N \in \mathbf{N}$. Уравнения такого рода, в зависимости от используемого локального времени - правого, левого или симметрического, обычно изучаются соответственно при следующих условиях на атомы: $\nu(\{x\})<1 / 2, \nu(\{x\})>-1 / 2$ и $|\nu(\{x\})|<1$. Эти условия позволяют свести уравнение с обобщенным сносом к уравнению без сноса и вывести условия сушествования и единственности решений из соответствующих результатов для уравнений без сноса. Основная цель настоящей статьи - исследовать случаи $\nu(\{x\}) \geqslant 1 / 2$, $\nu(\{x\}) \leqslant-1 / 2$ и $|\nu(\{x\})| \geqslant 1$ для некоторого $x \in \mathbf{R}$ и дать полное описание свойств уравнений с обобщенным сносом и их решений в этих случаях.

Ключевые слова и фразы: стохастические дифференциальные уравнения, локальные времена, обобщенный снос, отражение, поглощение, несуществование решений.

1. Introduction and basic definitions. Let $b$ be a measurable real function and $\nu$ a set function which is defined on the bounded Borel sets of the real line $\mathbf{R}$ such that it is a finite signed measure on $\mathscr{B}([-N, N])$ for every

${ }^{*}$ Finanz-DATA GmbH Beratungs- und Softwarehaus, Gotha, Germany; e-mail: s.blei@fida.de

${ }^{* *}$ Friedrich-Schiller-Universität Jena, Fakultät für Mathematik und Informatik, Institut für Stochastik, Jena, Germany; e-mail: hans-juergen.engelbert@uni-jena.de

1) This work was supported by the European Community's FP 7 Programme under contract PITN-GA-2008-213841, Marie Curie ITN «Controlled Systems». 
$N \in \mathbf{N}$. In the present note, we deal with the one-dimensional stochastic differential equation (SDE) with the so-called generalized drift introduced as

$$
X_{t}=X_{0}+\int_{0}^{t} b\left(X_{s}\right) \mathrm{d} B_{s}+\int_{\mathbf{R}} L^{X}(t, y) \nu(\mathrm{d} y),
$$

where $B$ is a Wiener process and $L^{X}$ denotes either the right, the left, or the symmetric local time of the unknown process $X$. We call $\nu$ appearing in equation (1.1) a drift measure.

SDEs of type (1.1) with generalized drift have been studied previously by many authors. We refer the reader to J. M. Harrison and L. A. Shepp [6], N. I. Portenko [10], D. W. Stroock and M. Yor [13], and J. F. Le Gall [8], [9]. H. J. Engelbert and W.Schmidt [4], [5] derived rather weak necessary and sufficient conditions on existence and uniqueness of solutions to SDEs with generalized drift. More recently, R. F. Bass and Z.-Q. Chen [1] also considered SDEs of type (1.1).

To treat the general equation (1.1) the additional assumption

$$
\left.\nu(\{x\})<\frac{1}{2} \quad \text { (respectively, } \nu(\{x\})>-\frac{1}{2},|\nu(\{x\})|<1\right), \quad x \in \mathbf{R},
$$

is posed on $\nu$ in case of considering the right (respectively, left, symmetric) local time (cf. [4], [5], [9], [13]). This condition allows to reduce (1.1) to an equation without drift, i.e., an equation of type (1.1), where the drift measure is the zero measure. Therefore, the well-known conditions on existence and uniqueness of solutions to equations without drift can be used to derive conditions on existence and uniqueness of solutions to equation (1.1) (see, e.g., [4], [5]). More precisely, under condition (1.2) the integral equation

$$
g(x)= \begin{cases}1-2 \int_{[0, x]} F(g, y) \nu(\mathrm{d} y), & x \geqslant 0, \\ 1+2 \int_{(x, 0)} F(g, y) \nu(\mathrm{d} y), & x<0\end{cases}
$$

where

$$
F(g, x)=g(x-) \quad\left(\text { respectively, } g(x), \frac{1}{2}(g(x)+g(x-))\right), \quad x \in \mathbf{R},
$$

admits a unique càdlàg solution. This solution $g$ is strictly positive, and the strictly increasing and continuous primitive $G(x)=\int_{0}^{x} g(y) \mathrm{d} y$ transforms (1.1) into an equation without drift (cf. [4, Proposition 1], or [5, Proposition (4.29)]). In the several cases, the explicit form of the solution to $(1.3)$ can be found in [5], (4.26), $\left(4.26^{\prime}\right)$, and $\left(4.26^{\prime \prime}\right)$, respectively.

The case $\nu(\{x\})=1 / 2$ (respectively, $\nu(\{x\})=-1 / 2,|\nu(\{x\})|=1$ ) for some $x \in \mathbf{R}$ is excluded in (1.3) since it corresponds, as we will see, to a reflecting barrier at the point $x$, which requires different methods to treat 
equation (1.1) than by assuming (1.2) (cf. [12], [1]). Moreover, in the case that $\nu(\{x\})>1 / 2$ (respectively, $\nu(\{x\})<-1 / 2,|\nu(\{x\})|>1$ ) holds for some $x \in \mathbf{R}$, in general, there is no solution to equation (1.1).

Indeed, in their famous paper on skew Brownian motion, J. M. Harrison and L. A. Shepp [6] studied equation (1.1) with symmetric local time in the special case $X_{0}=0, b \equiv 1$, and $\nu=\beta \delta_{0}$, where $\delta_{0}$ is the Dirac measure in zero, and they proved that there is no solution for the case $|\beta|>1$.

Referring to [6], J. F. Le Gall [9] (see after the proof of [9, Theorem 2.3]) asserted, without giving a proof, that in the context of [9], where $b$ is of finite variation and bounded from below by a strictly positive constant, the result on the nonexistence of a solution started at $x_{0}$ can be extended to equation (1.1) with symmetric local time if $\left|\nu\left(\left\{x_{0}\right\}\right)\right|>1$.

Also for symmetric local time, R. F. Bass and Z.-Q. Chen [1] stated some propositions if $|\nu(\{x\})|>1$ or $|\nu(\{x\})|=1$ for some $x \in \mathbf{R}$ under the assumption, besides others, that the diffusion coefficient $b$ is bounded from below by a positive constant (see [1, Theorems 3.2 and 3.3]). However, in both formulations and proofs, we feel that there is not enough clarity which had enabled us to follow their arguments. In particular, they have not pointed out where their assumptions on $b$ are used. But we shall see below that for general diffusion coefficients $b$ their Theorem 3.2 does not remain true.

The purpose of the present note is to give a general, complete and rigorous approach to equation (1.1) under the condition that, contrary to (1.2), for some $x \in \mathbf{R}$ the drift measure $\nu$ satisfies

$$
\nu(\{x\})>\frac{1}{2} \quad\left(\text { respectively, } \nu(\{x\})<-\frac{1}{2},|\nu(\{x\})|>1\right)
$$

or

$$
\left.\nu(\{x\})=\frac{1}{2} \quad \text { (respectively, } \nu(\{x\})=-\frac{1}{2},|\nu(\{x\})|=1\right) .
$$

The basic idea is to provide an insight into the behavior of the local times $L^{X}(t, x)$ of solutions $X$ of equation (1.1) in such points $x \in \mathbf{R}$ violating (1.2). This gives rise for an application of Tanaka's formula, followed by a space transformation, to conclude full information about the features of the solution.

Throughout the paper, $(\Omega, \mathscr{F}, \mathbf{P})$ stands for a complete probability space endowed with a filtration $\mathbb{F}=\left(\mathscr{F}_{t}\right)_{t \geqslant 0}$ which satisfies the usual conditions, i.e., $\mathbb{F}$ is right-continuous and $\mathscr{F}_{0}$ contains all sets from $\mathscr{F}$ which have P-measure zero. For a process $X=\left(X_{t}\right)_{t \geqslant 0}$ the notation $(X, \mathbb{F})$ indicates that $X$ is $\mathbb{F}$-adapted. The processes considered in the following belong to the class of continuous semimartingales up to a stopping time $S$ and local times of such processes will be an important tool. Given an $\mathbb{F}$-stopping time $S$, we say that $(X, \mathbb{F})$ is a semimartingale up to $S$ if there exists an increasing sequence $\left(S_{n}\right)_{n \in \mathbf{N}}$ of $\mathbb{F}$-stopping times such that $S=\lim _{n \rightarrow+\infty} S_{n}$ and, for 
every $n \in \mathbf{N}$, the process $\left(X^{S_{n}}, \mathbb{F}\right)$ obtained by stopping $(X, \mathbb{F})$ in $S_{n}$ is a real-valued semimartingale. Analogously, we introduce the notion of a local martingale up to $S$.

If $(X, \mathbb{F})$ is a semimartingale up to $S$, then we can find a decomposition

$$
X_{t}=X_{0}+M_{t}+V_{t}, \quad t<S, \quad \text { P-a.s., }
$$

on $[0, S)$, where $(M, \mathbb{F})$ is a local martingale up to $S$ with $M_{0}=0$ and $(V, \mathbb{F})$ is a right-continuous process whose paths are of bounded variation on $[0, t]$ for every $t<S$ and with $V_{0}=0$. If $X$ is continuous on $[0, S)$, then there exists a decomposition such that $M$ and $V$ are continuous on $[0, S)$ and this decomposition is unique on $[0, S)$. For any continuous local martingale $(M, \mathbb{F})$ up to $S$ by $\langle M\rangle$ we denote the continuous increasing process, which is uniquely determined on $[0, S)$, such that $\left(M^{2}-\langle M\rangle, \mathbb{F}\right)$ is a continuous local martingale up to $S$ and $\langle M\rangle_{0}=0$. For a continuous semimartingale $(X, \mathbb{F})$ up to $S$ we set $\langle X\rangle=\langle M\rangle$, where $M$ is the continuous local martingale up to $S$ in the decomposition (1.4) of $X$.

We now recall some facts which are well known for continuous semimartingales (see, for example, [11, Chap. VI, §1]). Their extension to semimartingales $(X, \mathbb{F})$ up to an $\mathbb{F}$-stopping time $S$ is obvious. For $(X, \mathbb{F})$ there exists the right (respectively, left, symmetric) local time $L^{X}$ up to $S$ which is a function on $[0, S) \times \mathbf{R}$ into $[0,+\infty)$ such that for every real function $f$ which is the difference of convex functions the generalized Itô formula holds:

$$
f\left(X_{t}\right)=f\left(X_{0}\right)+\int_{0}^{t} f^{\prime}\left(X_{s}\right) \mathrm{d} X_{s}+\frac{1}{2} \int_{0}^{t} L^{X}(t, y) \mathrm{d} f^{\prime}(y), \quad t<S, \quad \text { P-a.s. }
$$

where $f^{\prime}$ denotes the left (respectively, right, symmetric) derivative of $f$. To indicate explicitly which local time we consider, we write $L_{+}^{X}$ (respectively, $L_{-}^{X}, \widehat{L}^{X}$ ) for the right (respectively, left, symmetric) local time of $X$. If a formula or statement holds for every type of local time we just use the symbol $L^{X}$. Note that we can choose $L_{+}^{X}$ (respectively, $L_{-}^{X}$ ) to be increasing and continuous in $t<S$ and right- (respectively, left-) continuous with left- (respectively, right-) hand limits in $x$. Moreover, we have the relation $\widehat{L}^{X}=\left(L_{+}^{X}+L_{-}^{X}\right) / 2$. The local times fulfil the properties

$$
\begin{gathered}
\int_{0}^{t} \mathbf{1}_{\{y\}}\left(X_{s}\right) L^{X}(\mathrm{~d} s, y)=L^{X}(t, y), \quad t<S, \quad y \in \mathbf{R}, \quad \mathbf{P} \text {-a.s., } \\
L_{+}^{X}(t, y)-L_{-}^{X}(t, y)=2 \int_{0}^{t} \mathbf{1}_{\{y\}}\left(X_{s}\right) \mathrm{d} V_{s}, \quad t<S, \quad y \in \mathbf{R}, \quad \mathbf{P} \text {-a.s., } \\
L^{X}(t, y)=0, \quad t<S, \quad y \notin\left[\min _{0 \leqslant s \leqslant t} X_{s}, \max _{0 \leqslant s \leqslant t} X_{s}\right], \quad \mathbf{P} \text {-a.s., }
\end{gathered}
$$

and

$$
L^{X}(t, y)=\lim _{\varepsilon \downarrow 0} \frac{1}{\varepsilon} \int_{0}^{t} I_{\varepsilon}^{y}\left(X_{s}\right) \mathrm{d}\langle X\rangle_{s}, \quad t<S, y \in \mathbf{R}, \mathbf{P} \text {-a.s. }
$$


where

$$
I_{\varepsilon}^{y}(x)=\mathbf{1}_{[y, y+\varepsilon)}(x) \quad\left(\text { respectively, } \mathbf{1}_{(y-\varepsilon, y]}(x), \frac{1}{2} \mathbf{1}_{(y-\varepsilon, y+\varepsilon)}(x)\right), \quad x \in \mathbf{R} .
$$

In general, SDEs of type (1.1) admit exploding solutions. Therefore, the convenient state space is the extended real line $\overline{\mathbf{R}}=\mathbf{R} \cup\{-\infty,+\infty\}$ equipped with the $\sigma$-algebra $\mathscr{B}(\overline{\mathbf{R}})$ of Borel subsets. We fix the notion of a solution to equation (1.1) in the following

D e f i n i t i o n 1.1. A continuous $(\overline{\mathbf{R}}, \mathscr{B}(\overline{\mathbf{R}}))$-valued stochastic process $(X, \mathbb{F})$ defined on a probability space $(\Omega, \mathscr{F}, \mathbf{P})$ is called a solution to equation (1.1) if the following conditions are fulfilled:

(i) $X_{0}$ is real-valued;

(ii) $X_{t}=X_{t \wedge S_{\infty}^{X}}, t \geqslant 0, \mathbf{P}$-a.s., where $S_{\infty}^{X}:=\inf \left\{t \geqslant 0\right.$ : $\left.\left|X_{t}\right|=+\infty\right\}$;

(iii) $(X, \mathbb{F})$ is a semimartingale up to $S_{\infty}^{X}$;

(iv) there exists a Wiener process $(B, \mathbb{F})$ such that (1.1) is satisfied for all $t<S_{\infty}^{X} \mathbf{P}$-a.s.

2. The results. We recall that in (1.1) $L^{X}$ stands either for the right, the left or the symmetric local time and we treat these three cases simultaneously in the sequel. For the sake of brevity, in the following we write, for example, $\{\nu \geqslant 1 / 2\}$ instead of $\{y \in \mathbf{R}: \nu(\{y\}) \geqslant 1 / 2\}$.

Lemma 2.1. Let $(X, \mathbb{F})$ be a solution of equation (1.1) with right (respectively, left, symmetric) local time. Then it holds

$$
L_{-}^{X}(t, x)=0, \quad t<S_{\infty}^{X}, x \in\left\{\nu \geqslant \frac{1}{2}\right\}, \mathbf{P}-\text { a.s. }
$$

(respectively, $L_{-}^{X}(t, x)=0, \quad t<S_{\infty}^{X}, x \in\left\{\nu<-\frac{1}{2}\right\}, \mathbf{P}$-a.s.,

$$
\left.L_{-}^{X}(t, x)=0, \quad t<S_{\infty}^{X}, x \in\{|\nu|>1 \text { or } \nu=1\}, \mathbf{P} \text {-a.s. }\right)
$$

and

$$
L_{+}^{X}(t, x)=0, \quad t<S_{\infty}^{X}, x \in\left\{\nu>\frac{1}{2}\right\}, \mathbf{P} \text {-a.s. }
$$

(respectively, $L_{+}^{X}(t, x)=0, \quad t<S_{\infty}^{X}, x \in\left\{\nu \leqslant-\frac{1}{2}\right\}, \mathbf{P}$-a.s.,

$$
\left.L_{+}^{X}(t, x)=0, \quad t<S_{\infty}^{X}, x \in\{|\nu|>1 \text { or } \nu=-1\}, \mathbf{P} \text {-a.s. }\right) .
$$

P r o o f. Using (1.6) and (1.7), we see

$$
\begin{aligned}
L_{+}^{X}(t, x)-L_{-}^{X}(t, x) & =2 \int_{0}^{t} \mathbf{1}_{\{x\}}\left(X_{s}\right) \int_{\mathbf{R}} L^{X}(\mathrm{~d} s, y) \nu(\mathrm{d} y) \\
& =2 L^{X}(t, x) \nu(\{x\}), \quad t<S_{\infty}^{X}, \quad x \in \mathbf{R}, \quad \text { P-a.s. }
\end{aligned}
$$


and it follows

$$
\begin{aligned}
&(1-2 \nu(\{x\})) L_{+}^{X}(t, x)=L_{-}^{X}(t, x), \\
& \text { respectively, } \quad(1+2 \nu(\{x\})) L_{-}^{X}(t, x)=L_{+}^{X}(t, x), \\
&(1-\nu(\{x\})) L_{+}^{X}(t, x)=(1+\nu(\{x\})) L_{-}^{X}(t, x),
\end{aligned}
$$

$t<S_{\infty}^{X}, x \in \mathbf{R}, \mathbf{P}$-a.s., which, together with the nonnegativity of the local times, implies the claims. Lemma 2.1 is proved.

The following theorem is the main result of the present note.

Theorem 2.1. Let $(X, \mathbb{F})$ be a solution of equation (1.1) with right (respectively, left, symmetric) local time started at $x_{0} \in \mathbf{R}$. Then the following statements are satisfied.

(i) Assume $\nu\left(\left\{x_{0}\right\}\right) \geqslant 1 / 2$ (respectively, $\left.\nu\left(\left\{x_{0}\right\}\right) \leqslant-1 / 2,\left|\nu\left(\left\{x_{0}\right\}\right)\right| \geqslant 1\right)$. Then it holds for all $t \geqslant 0, \mathbf{P}$-a.s.

$$
X_{t} \geqslant x_{0},
$$

(respectively, $\quad X_{t} \leqslant x_{0}$,

$$
\left.X_{t} \leqslant x_{0}, \text { if } \nu\left(\left\{x_{0}\right\}\right) \leqslant-1 \text { and } X_{t} \geqslant x_{0} \text { if } \nu\left(\left\{x_{0}\right\}\right) \geqslant 1\right) \text {, }
$$

i.e., the point $x_{0}$ is reflecting.

(ii) Let $\nu\left(\left\{x_{0}\right\}\right)>1 / 2$ (respectively, $\left.\nu\left(\left\{x_{0}\right\}\right)<-1 / 2,\left|\nu\left(\left\{x_{0}\right\}\right)\right|>1\right)$. Then it holds

$$
X_{t}=x_{0}, \quad t \geqslant 0, \quad \mathbf{P} \text {-a.s. }
$$

i.e., the point $x_{0}$ is absorbing. In particular, $b\left(x_{0}\right)=0$ must be fulfilled.

$\mathrm{P}$ r o o f. 1) Let $(X, \mathbb{F})$ be a solution of equation (1.1) started at $x_{0} \in \mathbf{R}$. At first we reduce the problem to the case $x_{0}=0$. For the process $\left(X-x_{0}, \mathbb{F}\right)$ it clearly holds

$X_{t}-x_{0}=\int_{0}^{t} b_{x_{0}}\left(X_{s}-x_{0}\right) \mathrm{d} B_{s}+\int_{\mathbf{R}} L^{X-x_{0}}\left(t, y-x_{0}\right) \nu(\mathrm{d} y), \quad t<S_{\infty}^{X}, \quad \mathbf{P}$-a.s. where $b_{x_{0}}(x):=b\left(x+x_{0}\right), x \in \mathbf{R}$, and we have used the relation

$$
L^{X}(t, x)=L^{X-x_{0}}\left(t, x-x_{0}\right), \quad t<S_{\infty}^{X}, \quad x \in \mathbf{R}, \quad \text { P-a.s. }
$$

which can easily be deduced, for example, by exploiting (1.9). Introducing the drift measure $\nu_{x_{0}}$ via

$$
\nu_{x_{0}}(B):=\nu\left(B+x_{0}\right),{ }^{2)} \quad B \in \mathscr{B}([-N, N]), \quad N \in \mathbf{N},
$$

we obtain

$X_{t}-x_{0}=\int_{0}^{t} b_{x_{0}}\left(X_{s}-x_{0}\right) \mathrm{d} B_{s}+\int_{\mathbf{R}} L^{X-x_{0}}(t, y) \nu_{x_{0}}(\mathrm{~d} y), \quad t<S_{\infty}^{X}, \quad$ P-a.s.

2) For $B \subseteq \mathbf{R}$, we set $B+x_{0}:=\left\{x+x_{0}: x \in B\right\}$. 
Hence, $\left(X-x_{0}, \mathbb{F}\right)$ is also a solution to an equation of type (1.1) but started at zero and the drift measure satisfies $\nu_{x_{0}}(\{0\})=\nu\left(\left\{x_{0}\right\}\right)$. Therefore, without loss of generality we assume $x_{0}=0$ in the following parts of the proof.

2) Now let us consider the case of the right local time in equation (1.1). To prove (i) we assume $\nu(\{0\}) \geqslant 1 / 2$. Setting $Z_{t}=X_{t} \wedge 0, t \geqslant 0$, and applying Tanaka's formula for the left local time (see (1.5) for $f(x)=-x^{-}=x \wedge 0$, $x \in \mathbf{R}$ ) we obtain

$$
\begin{aligned}
Z_{t}=\int_{0}^{t} & \mathbf{1}_{(-\infty, 0)}\left(X_{s}\right) b\left(X_{s}\right) \mathrm{d} B_{s}+\int_{0}^{t} \mathbf{1}_{(-\infty, 0)}\left(X_{s}\right) \int_{\mathbf{R}} L_{+}^{X}(\mathrm{~d} s, y) \nu(\mathrm{d} y) \\
& -\frac{1}{2} L_{-}^{X}(t, 0), \quad t<S_{\infty}^{X}, \quad \mathbf{P} \text {-a.s. }
\end{aligned}
$$

Lemma 2.1 shows that the left local time of $X$ in zero vanishes and we can write

$$
\begin{aligned}
Z_{t} & =\int_{0}^{t} \mathbf{1}_{(-\infty, 0)}\left(Z_{s}\right) b\left(Z_{s}\right) \mathrm{d} B_{s}+\int_{\mathbf{R}} \int_{0}^{t} \mathbf{1}_{(-\infty, 0)}\left(X_{s}\right) L_{+}^{X}(\mathrm{~d} s, y) \nu(\mathrm{d} y) \\
& =\int_{0}^{t} \mathbf{1}_{(-\infty, 0)}\left(Z_{s}\right) b\left(Z_{s}\right) \mathrm{d} B_{s}+\int_{\mathbf{R}} L_{+}^{Z}(t, y) \mathbf{1}_{(-\infty, 0)}(y) \nu(\mathrm{d} y),
\end{aligned}
$$

$t<S_{\infty}^{X}$, P-a.s., where we used (1.6) and the easy to check relation $L_{+}^{X}(t, y)=$ $L_{+}^{Z}(t, y), t<S_{\infty}^{X}, y<0, \mathbf{P}$-a.s. (use, e.g., (1.9)). Introducing the sets

$$
A_{1}:=\left\{y \in(-\infty, 0): \nu(\{y\}) \geqslant \frac{1}{2}\right\}, \quad A_{2}:=\left\{y \in(-\infty, 0): \nu(\{y\})=\frac{1}{2}\right\}
$$

and the set function $\mu(\mathrm{d} y):=\mathbf{1}_{(-\infty, 0) \backslash A_{1}}(y) \nu(\mathrm{d} y)$, in the decomposition of $Z$ we can split the last integral and, using Lemma 2.1, we can write

$Z_{t}=\int_{0}^{t} \mathbf{1}_{(-\infty, 0)}\left(Z_{s}\right) b\left(Z_{s}\right) \mathrm{d} B_{s}+\int_{\mathbf{R}} L_{+}^{Z}(t, y) \mu(\mathrm{d} y)+\int_{\mathbf{R}} L_{+}^{Z}(t, y) \mathbf{1}_{A_{2}}(y) \nu(\mathrm{d} y)$,

$t<S_{\infty}^{X}$, P-a.s. Noting that $\mu(\{x\})<1 / 2, x \in \mathbf{R}$, we define the strictly positive function $g$ as a unique solution of the integral equation (1.3) with respect to $\mu$ and denote by $G(x)=\int_{0}^{x} g(y) \mathrm{d} y, x \in \overline{\mathbf{R}}$, its primitive which is strictly increasing and continuous. Note that $G$ restricted to $\mathbf{R}$ is the difference of convex functions. We use the notation

$$
M_{t}:=\int_{0}^{t} \mathbf{1}_{(-\infty, 0)}\left(Z_{s}\right) b\left(Z_{s}\right) \mathrm{d} B_{s}, \quad t<S_{\infty}^{X} .
$$

Then, due to the generalized Itô formula for the right local time and since $\mathrm{d} g(y)=-2 g(y-) \mu(\mathrm{d} y)$, for $Y=G(Z)$ it holds

$$
\begin{aligned}
Y_{t}= & \int_{0}^{t} g\left(Z_{s}\right) \mathrm{d} M_{s}+\int_{0}^{t} g\left(Z_{s}-\right) \int_{\mathbf{R}} L_{+}^{Z}(\mathrm{~d} s, y) \mu(\mathrm{d} y) \\
& +\int_{0}^{t} g\left(Z_{s}-\right) \int_{\mathbf{R}} L_{+}^{Z}(\mathrm{~d} s, y) \mathbf{1}_{A_{2}}(y) \nu(\mathrm{d} y)-\int_{\mathbf{R}} L_{+}^{Z}(t, y) g(y-) \mu(\mathrm{d} y) \\
= & \int_{0}^{t} g\left(Z_{s}\right) \mathrm{d} M_{s}+\int_{\mathbf{R}} L_{+}^{Z}(t, y) g(y-) \mathbf{1}_{A_{2}}(y) \nu(\mathrm{d} y),
\end{aligned}
$$


$t<S_{\infty}^{X}$, P-a.s.. Clearly, since we have $Z_{t} \leqslant 0, t \geqslant 0$, and since $G$ maps $(-\infty, 0]$ into $(-\infty, 0]$, it follows $Y_{t} \leqslant 0, t \geqslant 0$. Using that $g$ is strictly positive, we obtain

$$
\int_{0}^{t} g\left(Z_{s}\right) \mathrm{d} M_{s} \leqslant Y_{t} \leqslant 0, \quad t<S_{\infty}^{X}, \quad \mathbf{P} \text {-a.s. }
$$

Therefore, the process $\int_{0}^{\cdot} g\left(Z_{s}\right) \mathrm{d} M_{s}$ being a nonpositive continuous local martingale up to $S_{\infty}^{X}$ starting at zero must be zero $\mathbf{P}$-a.s. which implies $\int_{0}^{t} g^{2}\left(Z_{s}\right) \mathrm{d}\langle M\rangle_{s}=0, t<S_{\infty}^{X}$, P-a.s., and thus $M_{t}=0, t<S_{\infty}^{X}$, P-a.s. Hence, $Z$ is a process of locally bounded variation on $\left[0, S_{\infty}^{X}\right)$ and we obtain

$$
L_{ \pm}^{Z}(t, y)=0, \quad t<S_{\infty}^{X}, \quad y \in \mathbf{R}, \quad \mathbf{P} \text {-a.s. }
$$

Therefore, it holds $Z_{t}=0, t<S_{\infty}^{X}, \mathbf{P}$-a.s., which means $X_{t} \geqslant 0, t \geqslant 0$, $\mathbf{P}$-a.s. and (i) is proved.

3) Now, still considering the case of the right local time in equation (1.1), we show (ii) which is why we assume $\nu(\{0\})>1 / 2$. From (i) we derive $X_{t} \geqslant 0, t \geqslant 0, \mathbf{P}$-a.s., and hence via (1.8)

$$
X_{t}=\int_{0}^{t} b\left(X_{s}\right) \mathrm{d} B_{s}+\int_{\mathbf{R}} \mathbf{1}_{[0,+\infty)}(y) L_{+}^{X}(t, y) \nu(\mathrm{d} y), \quad t<S_{\infty}^{X}, \quad \mathbf{P} \text {-a.s. }
$$

Setting

$$
A_{1}:=\left\{y \in[0,+\infty): \nu(\{y\}) \geqslant \frac{1}{2}\right\}, \quad A_{2}:=\left\{y \in[0,+\infty): \nu(\{y\})=\frac{1}{2}\right\}
$$

and defining the set function $\mu(\mathrm{d} y):=\mathbf{1}_{[0,+\infty) \backslash A_{1}}(y) \nu(\mathrm{d} y)$, we can write

$$
X_{t}=\int_{0}^{t} b\left(X_{s}\right) \mathrm{d} B_{s}+\int_{\mathbf{R}} L_{+}^{X}(t, y) \mu(\mathrm{d} y)+\int_{\mathbf{R}} \mathbf{1}_{A_{2}}(y) L_{+}^{X}(t, y) \nu(\mathrm{d} y),
$$

$t<S_{\infty}^{X}$, P-a.s., since the right local time $L_{+}^{X}$ of $X$ vanishes on $A_{1} \backslash A_{2}$ by Lemma 2.1. Note that $\mu$ satisfies (1.2). Let $g$ be a unique solution of (1.3) with respect to $\mu$ and $G(x):=\int_{0}^{x} g(y) \mathrm{d} y, x \in \overline{\mathbf{R}}$, its primitive. Using similar arguments as in (2.1), for $Y:=G(X)$ we obtain

$$
Y_{t}=\int_{0}^{t} g\left(X_{s}\right) b\left(X_{s}\right) \mathrm{d} B_{s}+\int_{\mathbf{R}} g(y-) L_{+}^{X}(t, y) \mathbf{1}_{A_{2}}(y) \nu(\mathrm{d} y),
$$

$t<S_{\infty}^{X}, \mathbf{P}$-a.s.. Having in mind that $0 \notin A_{2}$ and that $\nu$ is a finite signed measure on $\mathscr{B}([-1,1])$, we conclude ${ }^{3)} c:=\inf A_{2}>0$. Therefore, the $\mathbb{F}$-stopping time $\tau_{c}:=\inf \left\{t \geqslant 0: X_{t}=c\right\}$ is strictly positive. For the stopped process $Y_{t}^{\tau_{c}}:=Y_{\tau_{c} \wedge t}, t \geqslant 0$, due to (1.8) it holds

$$
Y_{t}^{\tau_{c}}=\int_{0}^{\tau_{c} \wedge t} g\left(X_{s}\right) b\left(X_{s}\right) \mathrm{d} B_{s}, \quad t<S_{\infty}^{X}, \quad \mathbf{P} \text {-a.s. }
$$

3) $\inf \varnothing:=+\infty$. 
Since $G$ maps $[0,+\infty)$ into $[0,+\infty)$, the last relation means that $Y^{\tau_{c}}$ is a nonnegative continuous local martingale up to $S_{\infty}^{X}$ started at zero, which implies immediately $Y_{t}^{\tau_{c}}=0, t \geqslant 0, \mathbf{P}$-a.s. Hence, because of the obvious identity $\tau_{c}=\inf \left\{t \geqslant 0: Y_{t}=G(c)\right\}$ and $G(c)>0$, we conclude $\tau_{c}=+\infty$ $\mathbf{P}$-a.s. Finally, we obtain $X_{t}=0, t \geqslant 0, \mathbf{P}$-a.s. Moreover, we have

$$
0=\langle X\rangle_{t}=\int_{0}^{t} b^{2}\left(X_{s}\right) \mathrm{d} s=b^{2}(0) t, \quad t \geqslant 0, \quad \text { P-a.s. }
$$

which is only possible if $b(0)=0$.

4) Now we prove (i) and (ii) for the case of the left local time in equation (1.1). We recall that we only need to consider the initial value $x_{0}=0$. So, we treat the equation

$$
X_{t}=\int_{0}^{t} b\left(X_{s}\right) \mathrm{d} B_{s}+\int_{\mathbf{R}} L_{-}^{X}(t, y) \nu(\mathrm{d} y)
$$

where we assume $\nu(\{0\}) \leqslant-1 / 2$. Let $(X, \mathbb{F})$ be a solution of this equation. Introducing $\widetilde{b}(x)=b(-x), x \in \mathbf{R}$, and the Wiener process $\widetilde{B}=-B$, for $-X$ it holds

$$
-X_{t}=\int_{0}^{t} \widetilde{b}\left(-X_{s}\right) \mathrm{d} \widetilde{B}_{s}-\int_{\mathbf{R}} L_{-}^{X}(t, y) \nu(\mathrm{d} y), \quad t<S_{\infty}^{X}, \quad \text { P-a.s. }
$$

We define $\widetilde{\nu}(A):=-\nu(-A),{ }^{4)} A \in \mathscr{B}([-N, N]), N \in \mathbf{N}$. Then, since $S_{\infty}^{X}=S_{\infty}^{-X}$ and since $L_{-}^{X}(t, y)=L_{+}^{-X}(t,-y), t<S_{\infty}^{X}, y \in \mathbf{R}, \mathbf{P}$-a.s., which follows immediately from (1.9), we get

$$
-X_{t}=\int_{0}^{t} \widetilde{b}\left(-X_{s}\right) \mathrm{d} \widetilde{B}_{s}+\int_{\mathbf{R}} L_{+}^{-X}(t, y) \widetilde{\nu}(\mathrm{d} y), \quad t<S_{\infty}^{-X}, \quad \text { P-a.s. }
$$

Hence, $(-X, \mathbb{F})$ is a solution of an equation of type (1.1) with right local time started at zero and for the drift measure $\widetilde{\nu}$ it holds $\widetilde{\nu}(\{0\})=-\nu(\{0\}) \geqslant 1 / 2$. Using step 2) of the proof yields $X_{t} \leqslant 0, t<S_{\infty}^{X}, \mathbf{P}$-a.s. if $\nu(\{0\}) \leqslant-1 / 2$ and by step 3) we obtain $X_{t}=0, t<S_{\infty}^{X}$, P-a.s. as well as $b(0)=0$ if $\nu(\{0\})<-1 / 2$.

5) For the proof in case of the symmetric local time in equation (1.1) all tools are already presented above. But one must be careful in adapting them. We provide the idea but the details are left to the reader. Again without loss of generality we assume $x_{0}=0$. Let $(X, \mathbb{F})$ be a solution to

$$
X_{t}=\int_{0}^{t} b\left(X_{s}\right) \mathrm{d} B_{s}+\int_{\mathbf{R}} \widehat{L}^{X}(t, y) \nu(\mathrm{d} y) .
$$

We first assume that $|\nu(\{0\})|>1$ or $\nu(\{0\})=1$. Similarly to above in step 2) for $Z_{t}=X_{t} \wedge 0, t \geqslant 0$, we deduce

$$
Z_{t}=\int_{0}^{t} \mathbf{1}_{(-\infty, 0)}\left(Z_{s}\right) b\left(Z_{s}\right) \mathrm{d} B_{s}+\int_{\mathbf{R}} \widehat{L}^{Z}(t, y) \mathbf{1}_{(-\infty, 0)}(y) \nu(\mathrm{d} y),
$$

4) For $A \subseteq \mathbf{R}$, we set $-A:=\{-x: x \in A\}$. 
$t<S_{\infty}^{X}, \mathbf{P}$-a.s.. Furthermore, with the help of the sets

$$
\begin{gathered}
A_{1}:=\{y \in(-\infty, 0):|\nu(\{y\})| \geqslant 1\}, \quad A_{2}:=\{y \in(-\infty, 0): \nu(\{y\})=1\}, \\
A_{3}:=\{y \in(-\infty, 0): \nu(\{y\})=-1\},
\end{gathered}
$$

the set function $\mu(\mathrm{d} y):=\mathbf{1}_{(-\infty, 0) \backslash A_{1}}(y) \nu(\mathrm{d} y)$ and Lemma 2.1 , we can write

$$
\begin{aligned}
Z_{t}= & \int_{0}^{t} \mathbf{1}_{(-\infty, 0)}\left(Z_{s}\right) b\left(Z_{s}\right) \mathrm{d} B_{s}+\int_{\mathbf{R}} \widehat{L}^{Z}(t, y) \mu(\mathrm{d} y)+\frac{1}{2} \int_{A_{2}} L_{+}^{Z}(t, y) \nu(\mathrm{d} y) \\
& +\frac{1}{2} \int_{A_{3}} L_{-}^{Z}(t, y) \nu(\mathrm{d} y), \quad t<S_{\infty}^{X}, \quad \mathbf{P} \text {-a.s. }
\end{aligned}
$$

Using a unique solution $g$ of the integral equation (1.3) in case of the symmetric local time with respect to $\mu$, its primitive $G(x):=\int_{0}^{x} g(y) \mathrm{d} y, x \in \overline{\mathbf{R}}$, and setting $Y:=G(X)$, analogously as in (2.1) we get

$$
\begin{aligned}
Y_{t}= & \int_{0}^{t} g\left(Z_{s}\right) \mathrm{d} M_{s}+\frac{1}{2} \int_{A_{2}} L_{+}^{Z}(t, y) g(y) \nu(\mathrm{d} y) \\
& +\frac{1}{2} \int_{A_{3}} L_{-}^{Z}(t, y) g(y) \nu(\mathrm{d} y), \quad t<S_{\infty}^{X}, \quad \mathbf{P} \text {-a.s. }
\end{aligned}
$$

where $M:=\int_{0}^{s} \mathbf{1}_{(-\infty, 0)}\left(Z_{s}\right) b\left(Z_{s}\right) \mathrm{d} B_{s}$. Note that, because of $\mu(\{y\})=0$, $y \in A_{2} \cup A_{3}$, the function $g$ is continuous in the points of $A_{2} \cup A_{3}$. Since $\nu$ is a finite signed measure on $\mathscr{B}([-N, N]), N \in \mathbf{N}$, we deduce ${ }^{5)} c:=\sup A_{3}<0$ and hence the $\mathbb{F}$-stopping time

$$
\tau_{c}:=\inf \left\{t \geqslant 0: Z_{t}=c\right\}=\inf \left\{t \geqslant 0: Y_{t}=G(c)\right\}
$$

is strictly positive. Clearly, due to (1.8) we have

$$
Y_{t}=\int_{0}^{t} g\left(Z_{s}\right) \mathrm{d} M_{s}+\frac{1}{2} \int_{A_{2}} L_{+}^{Z}(t, y) g(y) \nu(\mathrm{d} y), \quad t<\tau_{c} \wedge S_{\infty}^{X}, \quad \text { P-a.s. }
$$

and similarly to (2.2) and the lines thereafter we conclude $Z_{t}=0, t<\tau_{c} \wedge S_{\infty}^{X}$, $\mathbf{P}$-a.s. By the definition of $\tau_{c}$ this gives $Z_{t}=0, t<S_{\infty}^{X}, \mathbf{P}$-a.s. and therefore $X_{t} \geqslant 0, t \geqslant 0, \mathbf{P}$-a.s.

Now we assume $|\nu(\{0\})|>1$ or $\nu(\{0\})=-1$. As shown in step 4$),-X$ is a solution of equation (1.1) with drift measure $\widetilde{\nu}$ defined by $\widetilde{\nu}(A)=-\nu(-A)$, $A \in \mathscr{B}([-N, N]), N \in \mathbf{N}$. Hence, from the result just proved we obtain $-X_{t} \geqslant 0, \geqslant 0, \mathbf{P}$-a.s. Summarizing, this yields

$$
\begin{gathered}
X_{t}=0, t \geqslant 0, \mathbf{P} \text {-a.s. and necessarily } b(0)=0 \text { if }|\nu(\{0\})|>1, \\
X_{t} \geqslant 0, t \geqslant 0, \mathbf{P} \text {-a.s. if } \nu(\{0\})=1,
\end{gathered}
$$

5) $\sup \varnothing:=-\infty$. 
and

$$
X_{t} \leqslant 0, t \geqslant 0, \mathbf{P} \text {-a.s. if } \nu(\{0\})=-1 \text {. }
$$

This finishes the proof of (i) and (ii) for the symmetric local time. Theorem 2.1 is proved.

Introducing the $\mathbb{F}$-stopping time $\tau_{x}:=\inf \left\{t \geqslant 0: X_{t}=x\right\}$ for a solution $(X, \mathbb{F})$ of equation $(1.1)$, we can state more generally the following corollary to Theorem 2.1.

Corollary 2.1. Let $(X, \mathbb{F})$ be a solution of equation (1.1) with right (respectively, left, symmetric) local time and arbitrary initial condition $X_{0}$. Then the following statements are satisfied.

(i) If $x \in \mathbf{R}$ is such that $\nu(\{x\}) \geqslant 1 / 2$ (respectively, $\nu(\{x\}) \leqslant-1 / 2$, $|\nu(\{x\})| \geqslant 1)$, then on $\left\{\tau_{x}<+\infty\right\}$ it holds

$$
\begin{aligned}
& X_{\tau_{x}+t} \geqslant x, \\
\text { (respectively, } \quad & X_{\tau_{x}+t} \leqslant x, \\
& \left.X_{\tau_{x}+t} \leqslant x \text { if } \nu(\{x\}) \leqslant-1 \text { and } X_{\tau_{x}+t} \geqslant x \text { if } \nu(\{x\}) \geqslant 1\right)
\end{aligned}
$$

for all $t \geqslant 0, \mathbf{P}$-a.s..

(ii) If $x \in \mathbf{R}$ is such that $\nu(\{x\})>1 / 2$ (respectively, $\nu(\{x\})<-1 / 2$, $|\nu(\{x\})|>1)$, then it holds

$$
X_{\tau_{x}+t}=x, \quad t \geqslant 0, \quad \mathbf{P} \text {-a.s. on }\left\{\tau_{x}<+\infty\right\} .
$$

In particular, if $\mathbf{P}\left(\left\{\tau_{x}<+\infty\right\}\right)>0$, then $b(x)=0$ must be fulfilled.

P r o o f. We only give the idea of the proof. The details are left to the reader. If $\mathbf{P}\left(\left\{\tau_{x}<+\infty\right\}\right)>0$ is satisfied, then we use the trace of

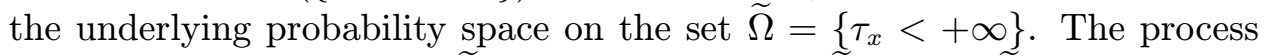
$X_{\tau_{x}+t}, t \geqslant 0$, considered on $\widetilde{\Omega}$, which is adapted to $\widetilde{\mathbb{F}}=\left(\mathscr{F}_{t} \cap \widetilde{\Omega}\right)_{t \geqslant 0}$, is again a solution of equation (1.1) but started at $x$. Hence, we can apply Theorem 2.1 to obtain the statements of the corollary.

Concerning the question of nonexistence of solutions of equation (1.1), we see from Theorem 2.1 and its Corollary 2.1 that the existence of a point $x \in \mathbf{R}$ with $\nu(\{x\})>1 / 2$ (respectively, $\nu(\{x\})<-1 / 2,|\nu(\{x\})|>1$ ), in general, does not imply automatically that there is no solution. In general, there can be solutions which do not reach $x$ or, if $b(x)=0$ is satisfied, which stay in $x$ after reaching this level. But it holds the following corollary.

Corollary 2.2. If $x \in \mathbf{R}$ is such that $\nu(\{x\})>1 / 2$ (respectively, $\nu(\{x\})<-1 / 2,|\nu(\{x\})|>1)$ and $b(x) \neq 0$, then there is no solution $(X, \mathbb{F})$ of equation (1.1) with right (respectively, left, symmetric) local time and arbitrary initial condition $X_{0}$ which satisfies $\mathbf{P}\left(\left\{\tau_{x}<+\infty\right\}\right)>0$. In particular, there is no solution started at $X_{0}=x$.

$\mathrm{R}$ e $\mathrm{m}$ a r k 2.1. (i) Considering the symmetric local time in equation (1.1), in the special case of a drift measure $\nu=\beta \delta_{0}$, where $|\beta|=1$ 
and $\delta_{0}$ denotes the Dirac measure in zero, the result of Corollary 2.1(i) was already presented in [2, Lemma 2.24]. Moreover, Corollary 2.2 contains [2, Lemma 2.25], which deals with the nonexistence of a solution to equation (1.1) with symmetric local time and drift measure $\nu=\beta \delta_{0}$ such that $|\beta|>1$.

(ii) In [12] one-dimensional stochastic differential equations with reflecting barriers, more precisely, equations of the form

(i) $X_{t}=X_{0}+\int_{0}^{t} b\left(X_{s}\right) \mathrm{d} B_{s}+L_{t}-R_{t}$

(ii) $X_{t} \in\left[r_{1}, r_{2}\right]$,

(iii) $L_{t}, R_{t}$ are increasing processes with $L_{0}=R_{0}=0$ and

$$
\int_{0}^{t} \mathbf{1}_{\left\{r_{1}\right\}}\left(X_{s}\right) \mathrm{d} L_{s}=L_{t}, \quad \int_{0}^{t} \mathbf{1}_{\left\{r_{2}\right\}}\left(X_{s}\right) \mathrm{d} R_{s}=R_{t}, \quad t \geqslant 0,
$$

where $r_{1}<r_{2}$, were studied. We recall that $(X, \mathbb{F})$ on $(\Omega, \mathscr{F}, \mathbf{P})$ is called a solution of $(2.3)$ if there exist a Wiener process $(B, \mathbb{F})$ and two processes $(L, \mathbb{F})$ and $(R, \mathbb{F})$ such that $(2.3)$ is satisfied. For a solution $(X, \mathbb{F})$ of equation (2.3) it is not difficult to check that $L$ and $R$ are just the symmetric local times of $X$ in $r_{1}$ and $r_{2}$, respectively. Our results, especially Corollary 2.1(i), now show that equation (2.3) even coincides with equation (1.1) with diffusion coefficient $b$, symmetric local time, drift measure $\nu=\delta_{r_{1}}-\delta_{r_{2}}$ and initial condition $X_{0} \in\left[r_{1}, r_{2}\right]$, i.e., with the equation

$$
X_{t}=X_{0}+\int_{0}^{t} b\left(X_{s}\right) \mathrm{d} B_{s}+\widehat{L}^{X}\left(t, r_{1}\right)-\widehat{L}^{X}\left(t, r_{2}\right), \quad X_{0} \in\left[r_{1}, r_{2}\right]
$$

For equation (2.4) the condition (2.3)(ii) need not be specified since it is satisfied for any solution which follows via Corollary 2.1(i). Moreover, (2.3)(iii) holds because of (1.6).

(iii) Similarly to the preceding remark the nonnegativity condition $X_{t} \geqslant 0, t \geqslant 0$, of the solution to the equation

$$
X_{t}=X_{0}+\int_{0}^{t} \mathbf{1}_{(0,+\infty)}\left(X_{s}\right) \mathrm{d} B_{s}+a \int_{0}^{t} \mathbf{1}_{\{0\}}\left(X_{s}\right) \mathrm{d} s
$$

with $X_{0} \geqslant 0$ and $a \geqslant 0$, which is studied in [3] (also see the recent paper [7]), can be dropped. More detailed, for a solution $(X, \mathbb{F})$ of this equation by (1.9) it follows $L_{-}^{X}(t, 0)=0, t \geqslant 0, \mathbf{P}$-a.s. Combined with (1.7) this implies

$$
\frac{1}{2} L_{+}^{X}(t, 0)=a \int_{0}^{t} \mathbf{1}_{\{0\}}\left(X_{s}\right) \mathrm{d} s, \quad t \geqslant 0, \quad \mathbf{P} \text {-a.s. }
$$

Hence, $(X, \mathbb{F})$ also solves equation (1.1) with diffusion coefficient $b=\mathbf{1}_{(0,+\infty)}$, right local time and drift measure $\nu=\delta_{0} / 2$. Therefore, we can conclude $X_{t} \geqslant 0, t \geqslant 0, \mathbf{P}$-a.s. if $X_{0} \geqslant 0$. 
With the help of Lemma 2.1 we can also give a relation between the different versions of equation (1.1). More precisely, we give a relation between equation (1.1) using the right local time and equation (1.1) with symmetric local time. With the following proposition we complement [1], Theorem $2.2(\mathrm{a})$.

Proposition 2.1. (i) $(X, \mathbb{F})$ is a solution of equation (1.1) with right local time and drift measure $\nu$ if and only if it is a solution of equation (1.1) with symmetric local time and drift measure

$$
\hat{\nu}(\mathrm{d} y)=\left(\frac{1}{2-2 \nu(\{y\})} \mathbf{1}_{\{z \in \mathbf{R}: 2 \nu(\{z\})<1\}}(y)+\mathbf{1}_{\{z \in \mathbf{R}: 2 \nu(\{z\}) \geqslant 1\}}(y)\right) 2 \nu(\mathrm{d} y) .
$$

(ii) $(X, \mathbb{F})$ is a solution of equation (1.1) with symmetric local time and drift measure $\hat{\nu}$ satisfying $\hat{\nu}(\{x\}) \neq-1, x \in \mathbf{R}$, if and only if it is a solution of equation (1.1) with right local time and drift measure

$$
\nu(\mathrm{d} y)=\left(\frac{2}{1+\hat{\nu}(\{y\})} \mathbf{1}_{\{z \in \mathbf{R}: \hat{\nu}(\{z\})>-1\}}(y)-\mathbf{1}_{\{z \in \mathbf{R}: \hat{\nu}(\{z\})<-1\}}(y)\right) \frac{1}{2} \widehat{\nu}(\mathrm{d} y) .
$$

P r o o f. 1) Let $(X, \mathbb{F})$ be a solution of equation (1.1) with right local time, then by Lemma 2.1

$\widehat{L}^{X}(t, y)=\frac{1}{2}\left(L_{+}^{X}(t, y)+L_{-}^{X}(t, y)\right)=0, \quad t<S_{\infty}^{X}, \quad y \in\left\{\nu>\frac{1}{2}\right\}, \quad \mathbf{P}$-a.s.

and

$$
\begin{gathered}
\widehat{L}^{X}(t, y)=\frac{1}{2} L_{+}^{X}(t, y)=(1-\nu(\{y\})) L_{+}^{X}(t, y), \\
t<S_{\infty}^{X}, \quad y \in\left\{\nu=\frac{1}{2}\right\}, \quad \mathbf{P} \text {-a.s. }
\end{gathered}
$$

Moreover, via (1.7) we see

$L_{+}^{X}(t, y)-L_{-}^{X}(t, y)=2 L_{+}^{X}(t, y) \nu(\{y\}), \quad t<S_{\infty}^{X}, \quad y \in\left\{\nu<\frac{1}{2}\right\}, \quad \mathbf{P}$-a.s.

and we can conclude

$$
\widehat{L}^{X}(t, y)=(1-\nu(\{y\})) L_{+}^{X}(t, y), \quad t<S_{\infty}^{X}, \quad y \in\left\{\nu<\frac{1}{2}\right\}, \quad \text { P-a.s. }
$$

Summarizing, we obtain that $(X, \mathbb{F})$ fulfils equation (1.1) with symmetric local time and drift measure $\hat{\nu}$ as given under (i).

2 ) If $(X, \mathbb{F})$ is a solution of equation (1.1) with symmetric local time and drift measure $\hat{\nu}$, then Lemma 2.1 implies

$$
L_{ \pm}^{X}(t, y)=\widehat{L}^{X}(t, y)=0, \quad t<S_{\infty}^{X}, \quad y \in\{|\hat{\nu}|>1\}, \quad \text { P-a.s. }
$$

and

$$
L_{-}^{X}(t, y)=0, \quad t<S_{\infty}^{X}, \quad y \in\{\hat{\nu}=1\}, \quad \text { P-a.s. }
$$


Furthermore, via (1.7) we obtain

$$
L_{+}^{X}(t, y)-L_{-}^{X}(t, y)=2 \widehat{L}^{X}(t, y) \widehat{\nu}(\{y\}), \quad t<S_{\infty}^{X}, \quad y \in \mathbf{R}, \quad \text { P-a.s. },
$$

and hence

$$
L_{+}^{X}(t, y)=(1+\hat{\nu}(\{y\})) \widehat{L}^{X}(t, y), \quad t<S_{\infty}^{X}, \quad y \in\{|\hat{\nu}|<1\}, \quad \text { P-a.s. }
$$

These observations mean, if we additionally assume $\hat{\nu}(\{x\}) \neq-1, x \in \mathbf{R}$, that $(X, \mathbb{F})$ satisfies (1.1) with right local time and drift measure $\nu$ as given in (ii).

3) Note that $\hat{\nu}$ as defined in (i) satisfies $\hat{\nu}(\{x\})>-1, x \in \mathbf{R}$, and it holds $\hat{\nu}(\{x\})>1$ if and only if $\nu(\{x\})>1 / 2$. Hence, the remaining part of assertion (i) follows by an application of step 2) of the proof. To prove the sufficiency in (ii), we remark that $\nu$ introduced in (ii) satisfies $\nu(\{x\})>1 / 2$ if and only if $|\hat{\nu}(\{x\})|>1$ and we can conclude using step 1) above. Proposition 2.1 is proved.

R e m a r k 2.2. (i) The drift measures $\hat{\nu}$ and $\nu$ as defined in Proposition 2.1(i) and (ii), respectively, are of course not unique.

(ii) Proposition 2.1(ii) gives an alternative to conclude the results of Theorem 2.1 and its Corollary 2.1 and 2.2 for equation (1.1) with symmetric local time and a drift measure satisfying $\nu(\{x\}) \neq-1, x \in \mathbf{R}$, from the results for the right local time.

(iii) The case $\hat{\nu}(\{x\})=-1$ for some $x \in \mathbf{R}$ is excluded in Proposition 2.1(ii), since this condition is responsible for reflection to the left, but this cannot occur for solutions of equation (1.1) where the right local time is chosen: By Lemma 2.1 we then have $L_{+}^{X}(t, x)=0$.

Similar conclusions as in Proposition 2.1 can be made when the left local time is involved. For the sake of completeness we state the corresponding results which can be proved by analogous arguments as before.

Proposition 2.2. (i) $(X, \mathbb{F})$ is a solution of (1.1) with right local time and drift measure $\nu$ satisfying $\nu(\{x\}) \neq 1 / 2, x \in \mathbf{R}$, if and only if it is a solution of equation (1.1) with left local time and drift measure

$$
\bar{\nu}(\mathrm{d} y)=\left(\frac{1}{1-2 \nu(\{y\})} \mathbf{1}_{\{z \in \mathbf{R}: \nu(\{z\})<1 / 2\}}(y)-\mathbf{1}_{\{z \in \mathbf{R}: \nu(\{z\})>1 / 2\}}(y)\right) \nu(\mathrm{d} y) .
$$

(ii) $(X, \mathbb{F})$ is a solution of equation (1.1) with left local time and drift measure $\bar{\nu}$ satisfying $\bar{\nu}(\{x\}) \neq-1 / 2, x \in \mathbf{R}$, if and only if it is a solution of equation (1.1) with right local time and drift measure

$$
\nu(\mathrm{d} y)=\left(\frac{1}{1+2 \bar{\nu}(\{y\})} \mathbf{1}_{\{z \in \mathbf{R}: 2 \bar{\nu}(\{z\})>-1 / 2\}}(y)+\mathbf{1}_{\{z \in \mathbf{R}: 2 \bar{\nu}(\{z\})<-1 / 2\}}(y)\right) \bar{\nu}(\mathrm{d} y) .
$$

(iii) $(X, \mathbb{F})$ is a solution of equation (1.1) with left local time and drift measure $\bar{\nu}$ if and only if it is a solution of equation (1.1) with symmetric 
local time and drift measure

$$
\hat{\nu}(\mathrm{d} y)=\left(\frac{1}{2+2 \bar{\nu}(\{y\})} \mathbf{1}_{\{z \in \mathbf{R}: \bar{\nu}(\{z\})>-1\}}(y)+\mathbf{1}_{\{z \in \mathbf{R}: \bar{\nu}(\{z\}) \leqslant-1\}}(y)\right) 2 \bar{\nu}(\mathrm{d} y) .
$$

(iv) $(X, \mathbb{F})$ is a solution of equation (1.1) with symmetric local time and drift measure $\hat{\nu}$ satisfying $\hat{\nu}(\{x\}) \neq 1, x \in \mathbf{R}$, if and only if it is a solution of equation (1.1) with left local time and drift measure

$$
\bar{\nu}(\mathrm{d} y)=\left(\frac{2}{1-\hat{\nu}(\{y\})} \mathbf{1}_{\{z \in \mathbf{R}: \hat{\nu}(\{z\})<1\}}(y)-\mathbf{1}_{\{z \in \mathbf{R}: \hat{\nu}(\{z\})>1\}}(y)\right) \frac{1}{2} \hat{\nu}(\mathrm{d} y) .
$$

\section{REFERENCES}

1. Bass R.F., Chen Z.-Q. One-dimensional stochastic defferential equations with singular and degenerate coefficients. - Sankhyā, 2005, v. 67, № 1, p. 19-45.

2. Blei S. On symmetric and skew Bessel processes. - Stochastic Process. Appl., 2012, v. 122, № 9, p. 3262-3287.

3. Chitashvili $R$. On the nonexistence of a strong solution in the boundary problem for a sticky Brownian motion. - Proc. A. Razmadze Math. Inst., 1997, v. 115, p. 17-31.

4. Engelbert H. J., Schmidt W. On one-dimensional stochastic differential equations with generalized drift. - Lecture Notes in Control and Inform. Sci., 1985, v. 69, p. 143-155.

5. Engelbert H. J., Schmidt W. Strong Markov continuous local martingales and solutions of the one-dimensional stochastic differential equations. III. - Math. Nachr., 1991, v. 151 , p. $149-197$.

6. Harrison J. M., Shepp L. A. On skew Brownian motion. - Ann. Probab., 1981, v. 9, № 2, p. 309-313.

7. Karatzas I., Shiryaev A.N., Shkolnikov M. On the one-sided Tanaka equation, with drift. - Electron Commun. Probab., 2011, v. 16, p. 664-677.

8. Le Gall J.-F. Applications du temps local aux équations différentielles stochastiques unidimensionnelles. - Lecture Notes in Math., 1983, v. 986, p. 15-31.

9. Le Gall J.-F. One-dimensional stochastic differential equations involving the local times of the unknown process. - Lecture Notes in Math., 1984, v. 1095, p. 51-82.

10. Портенко Н.И. Обобщенные диффузионные процессы. Киев: Наукова думка, 1982, $208 \mathrm{c.}$

11. Revuz D., Yor M. Continuous Martingales and Brownian Motion. Berlin: SpringerVerlag, 1999, 602 p. (Grundlehren Math. Wiss., v. 293.)

12. Schmidt $W$. On stochastic differential equations with reflecting barries. - Math. Nachr., 1989, v. 142, p. 135-148.

13. Stroock D. W., Yor M. Some remarkable martingales. - Lecture Notes in Math., 1981 , v. 850 , p. $590-603$.

Поступила в редакцию 15.VIII.2012

Исправленный вариант 25.VII.2013 